\title{
PFLEGE
}

\section{Bürokratische Überregulierung}

\author{
Michael Graber-Dünow
}

\begin{abstract}
Als Beispiel für die wachsende Bürokratisierung in der Sozialwirtschaft wird immer wieder die stationäre Altenhilfe genannt.
\end{abstract}

Die wachsende Bürokratisierung in der stationären Altenhilfe wird in der Fachöffentlichkeit seit einigen Jahren thematisiert. Wie der Verband Deutscher Alten- und Behindertenhilfe errechnete, können mittlerweile nur noch 62 Prozent der Arbeitszeit von Pflegenden für die praktische Pflege erbracht werden. (1) Der von den zuständigen Bundesministerien eingerichtete »Runde Tisch Pflege« beschäftigte sich daher auch in einer Arbeitsgruppe einzig mit notwendigen Maßnahmen zur »Entbürokratisierung «; die Ergebnisse der Arbeitsgruppe liegen seit September 2005 vor. (2)

\section{Externe Qualitätskontrollen}

Heime müssen mehr als 980 bundesweit geltende Rechtsvorschriften beachten (3), deren Einhaltung von verschiedenen Behörden und Prüfinstanzen wie Heimaufsicht, Medizinischer Dienst der Krankenkassen, Gesundheitsamt, vorbeugender Brandschutz, Bauaufsicht, Vormundschaftsgericht oder Berufsgenossenschaft überprüft werden.

Dabei kommt es nicht nur zu Überschneidungen der Prüfaufträge, sondern teilweise sogar zu sich widersprechenden Auflagen. So kann beispielsweise die Heimaufsichtsbehörde aus milieutherapeutischen Gründen die Gestaltung der Flurenden als »sekundäre Wohnbereiche« fordern, während die Feuerwehr auf die Entfernung jeglicher »Brandlast « und damit aller Möbel aus den Fluren besteht. Da die Heime nicht beide Auflagen erfüllen können, werden sie zwischen solch divergierenden Anforderungen der Aufsichtsbehörden zerrieben.

Der »Runde Tisch Pflege« empfiehlt daher auch »die Prüfung der Pflegeeinrichtungen zu den Feldern Hygiene, Brandschutz, Arbeitsschutz und Sicherheitstechnik sowie Trinkwasser bei einer Behörde zusammenzufassen oder unter der Verantwortung der Heimaufsicht zumindest eine Koordination herbeizuführen«. Dies könnte sicherlich ein erster Schritt sein, um in den genannten Bereichen zeitund kostenintensive Doppelprüfungen mit sich eventuell sogar widersprechenden Auflagen zu vermeiden.

\section{MDK und Heimaufsicht}

Am weitestgehenden sind die Überschneidungen der Prüfaufträge zwischen der Aufsichtsbehörde nach dem Heimgesetz und dem Medizinischen Dienst der Kranken-

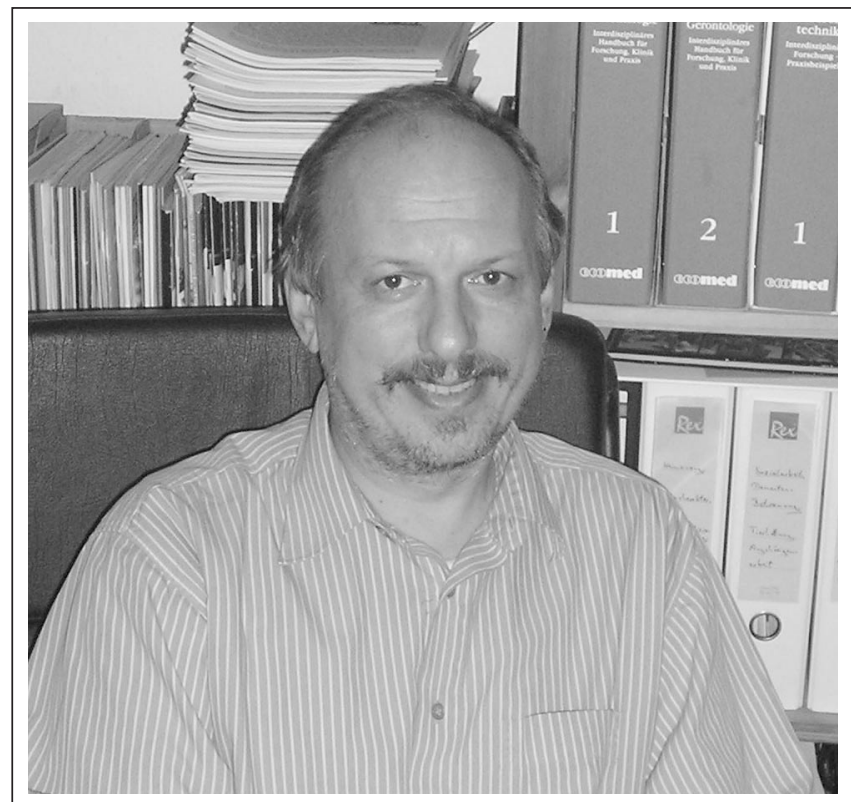

Michael Graber-Dünow (48) ist Diplom-Sozialarbeiter und staatlich anerkannter Altenpfleger. Nach Tätigkeiten in beiden Berufen war er ab 1988 im Leitungsteam eines großen Altenhilfezentrums beschäftigt. Seit 1997 ist er Leiter der Altenpflegeheims Justina von Cronstetten Stift in Frankfurt am Main (Internet http://www.justina-von-cronstetten-stift.de). Michael Graber-Dünow ist außerdem Fachbuchautor und im Aus- und Fortbildungsbereich, $u$. a. als Lehrbeauftragter im Studiengang Sozialarbeit der Frankfurter Fachhochschule, tätig.

kassen (MDK). Der Gesetzgeber hat dieses Problem zwar erkannt und sowohl im Pflegequalitätssicherungsgesetz wie auch mit der Novellierung des Heimgesetzes die beiden Institutionen zur Zusammenarbeit verpflichtet, um Doppelprüfungen nach Möglichkeit zu vermeiden. Doch die Praxis zeigt, dass dieses Problem dadurch noch lange nicht gelöst ist.

Auch der »Runde Tisch Pflege« hat in seinen Empfehlungen zur Entbürokratisierung eine »Harmonisierung im System öffentlich-rechtlicher Prüfungen « empfohlen: Die Inhalte der Prüfungen durch Heimaufsicht und MDK sollten danach eindeutig abgegrenzt und aufeinander abgestimmt werden. Ob dies tatsächlich in die Praxis umgesetzt wird und damit zu einer Reduzierung des bürokratischen Aufwands führt, darf jedoch bezweifelt werden. Auch die konkreten Vorschläge, die der »Runde 
Tisch Pflege « dazu selbst unterbreitet, sind zu diffus, um eine praxistaugliche Abgrenzung zu gewährleisten. So soll beispielsweise die Heimaufsicht für die »hauswirtschaftliche Versorgung und Verpflegung « zuständig sein, während der MDK die »Ergebnisqualität im Bereich Ernährung « überprüfen soll. Die Schnittmenge dieser beiden Prüfaufträge ist aber so groß, dass zwangsläufig doch wieder Doppelprüfungen entstehen werden.

Das grundlegende Problem besteht darin, dass die verschiedenen Behörden eine Legimitation ihrer eigenen Existenz benötigen. Es ist daher kaum davon auszugehen, dass sie bereit sein werden, Aufgabenbereiche an andere abzutreten.

So sieht beispielsweise die ab Januar 2006 gültige MDKPrüfrichtlinie als einen neuen Schwerpunkt die Prüfung der »sozialen Betreuung « im Heim vor und somit einem Bereich, der bisher zum Terrain der Heimaufsichtsbehörde zählte. (4) Da aber zu erwarten ist, dass diese kaum freiwillig auf die Kontrolle dieses Bereichs verzichten wird, ist für die Zukunft sogar mit einer Zunahme von Doppelprüfungen zu rechnen. In diesem Zusammenhang muss zugleich angemerkt werden, dass die fachliche Kompetenz zur Überprüfung der sozialen Betreuung beim MDK überhaupt nicht gegeben ist. We-

der die dort beschäftigten Ärzte noch die Pflegekräfte sind fachlich in der Lage, beispielweise die Arbeit eines Sozialdienstes kompetent zu bewerten. Die einseitig durch den MDK erfolgte Vereinnahmung dieses Prüfauftrags wird daher sicherlich nicht zu einer Verbesserung der Betreuungsqualität in den Heimen führen, sondern es bleibt eher zu befürchten, dass die Heime durch die zu erwartenden weiteren Doppelprüfungen mit zusätzlichem bürokratischen Aufwand belastet werden.

\section{Unabhängige Prüfinstanzen schaffen}

Da die Heimaufsicht auf Länderebene organisiert ist, gibt es zwischen den einzelnen Bundesländern zweifellos große Unterschiede hinsichtlich der Ausstattung und fachlichen Kompetenz dieser Behörden. Als wegweisend für die Organisation der Heimaufsicht kann jedoch das Bundesland Hessen gelten: Die Heimaufsicht ist dort der Versorgungsverwaltung angegliedert, also einer Behörde, die im Heimbereich von Kostenträger- und sonstigen Interessen unabhängig ist. Eine solche Unabhängigkeit ist nicht in allen Bundesländern gegeben, da die mit der Heimaufsicht beauftragten Behörden teilweise zugleich Kostenträger und/oder Heimträger sind. Auch der MDK ist letztlich ein Organ der Pflegekassen und somit eines Kostenträgers, der im Heimbereich spezifische Interessen verfolgt.

Mit einem Juristen als Dezernenten, Altenpflegern, Sozialarbeitern und Verwaltungsfachkräften verfügen die hessischen Heimaufsichtsbehörden über ein multiprofessionelles Team, das die Prüfungen nach dem Heimgesetz kompetent und sachbezogen durchführt. Es stellt sich daher die Frage, ob es nicht sinnvoller wäre, die bisherigen Doppelprüfungen durch Heimaufsicht und MDK gänzlich abzuschaffen und die Kontrolle der Pflege- und Betreuungsqualität der Heime in einer Aufsichtsbehörde zu bündeln, für deren Organisation die Hessische Heimaufsicht als Vorbild dienen könnte. Leider hat sich der »Runde Tisch Pflege « nicht für eine so weitgehende und radikale Lösung der Problematik ausgesprochen.

Einen erfreulichen Schritt in Richtung der anzustrebenden Harmonisierung der Prüfungen stellt aber immerhin die Ankündigung des MDK dar, künftig auf die Kontrollen der baulichen Ausstattung der Heime und der Mitwirkung des Heimbeirates zu verzichten. Allerdings sei dazu angemerkt, dass der MDK diese Prüfungen bisher durchführte, ohne überhaupt über eine entsprechende Rechtsgrundlage zu verfügen.

\section{Internes Qualitätsmanagement}

Neben den externen Prüfungen müssen die Heime nach dem Pflegequalitätssicherungsgesetz ein einrichtungsinternes Qualitätsmanagement implementieren. Die Forderung nach einem an den Bedürfnissen der Bewohner orientierten kontinuierlichen Verbesserungsprozess der

\section{»Heime müssen mehr als 980 bundesweit geltende Rechts- vorschriften beachten «}

Leistungen des Heimes ist fachlich begrüßenswert. Ein Umsetzungsproblem besteht allerdings darin, dass dies mit einer unveränderten personellen Ausstattung zu erfolgen hat. Qualitätsmanagement ist jedoch eine zusätzliche Aufgabe, die erhebliche zeitliche und personelle Ressourcen bindet und daher auch seitens der Kostenträger durch die Genehmigung zusätzlicher Planstellen abgesichert werden müsste. Die mit dem Qualitätsmanagement verbundenen Aufgaben werden derzeit jedoch von den Pflegenden (und anderen Mitarbeitern des Heimes) zusätzlich zu ihren ohnehin zeitlich kaum zu bewältigenden Arbeitsinhalten und damit letztlich zulasten der praktischen Pflege ausgeführt. Manche Träger haben sogar Planstellen im Pflegebereich abgebaut, um relativ gut dotierte Stellen im Qualitätsmanagement zu schaffen. Auch dies führt keinesfalls zu der eigentlich intendierten Qualitätsverbesserung.

Des Weiteren ist in diesem Zusammenhang festzustellen, dass viele der im Heimbereich gängigen Qualitätssicherungsinstrumente leider wenig geeignet sind, die Pflege- und Betreuungsqualität tatsächlich zu sichern und weiterzuentwickeln, da sie sich lediglich auf Formalismen, Abläufe und administrative Handlungen beziehen.

Dies beruht auf einem administrativ-technischen Verständnis dieser Instrumente, die vielfach entwickelt wurden, um industrielle Produktionsabläufe zu optimieren. Eine Übertragung von Industrienormen auf eine von einem ganzheitlichen Menschenbild geleitete Pflege alter Menschen kann dieser aber in keiner Weise gerecht werden. 
Bewohnerorientierte Verfahren, wie das englische Modell »Homes are for living in « (5) fristen in der deutschen Altenhilfepraxis ein Schattendasein. Auch ein Qualitätssiegel oder Qualitätszertifikat, wie es zunehmend von Einrichtungen angestrebt wird, sagt daher nichts über die tatsächliche Qualität der Pflege aus, sondern kann den Einrichtungen bestenfalls als teuer bezahltes Marketinginstrument dienen.

Der »Runde Tisch Pflege« bestätigt diese Einschätzung und empfiehlt daher die Erarbeitung eines »Anforderungskatalogs «, in dem die »Qualitätsanforderungen für die Arbeitsbereiche Pflege, Hauswirtschaft, soziale Betreuung, Gebäude und Organisation klar definiert werden«.

\section{Problem Pflegedokumentation}

Zweifellos sind Pflegedokumentation und Pflegeplanung für eine qualitätsgeleitete bewohnerorientierte $\mathrm{Al}$ tenpflege unverzichtbar. Trotzdem entstehen in diesem Zusammenhang zwei Problembereiche, die letztlich sogar zu einer Minderung der Pflegequalität führen können: Die sukzessive Einführung von Pflegedokumentation und Pflegeplanung vor etwa 20 Jahren stellte eine zusätzliche Aufgabe für die Pflegenden dar, ohne dass eine entsprechende Kompensation durch zusätzliche Personalstellen erfolgte.

Mit Einführung der Pflegeversicherung nahm die personelle Ausstattung der Heime - bei gleichzeitigem Anstieg der Pflegeintensität der Bewohner - sogar tendenziell ab. Da die Einführung von Pflegedokumentation und Pflegeplanung also letztlich zulasten der praktischen Pflege erfolgte, wurden und werden sie von manchen Pflegekräften noch immer als zusätzliche Belastung empfunden. Wie schon bei der Problematik der Implementierung eines internen Qualitätsmanagements sind auch hier die Kostenträger aufgefordert, einen Ausgleich durch die Bewilligung zusätzlicher Planstellen zu schaffen.

Das zweite Problem besteht darin, dass die Anforderungen an Pflegedokumentation und Pflegeplanung nicht einheitlich definiert, sondern dem Gutdünken der einzelnen Prüfer der verschiedenen Aufsichtsbehörden überlassen sind. Wie die Praxis zeigt, werden hier teilweise so weit gehende Anforderungen gestellt, dass Pflegedokumentation und Pflegeplanung von einem unverzichtbaren Instrument der Qualitätssicherung zuweilen zum reinen Selbstzweck zu verkommen droht.

Darüber hinaus sind die von manchen Prüfern geforderten umfassenden Datenerhebungen über die Heimbewohner auch ethisch höchst problematisch. Ein "gläserner Bewohner «, dessen intimste Regungen und Geheimnisse vom Personal in »Dossiers « festgehalten werden, erinnert wieder fatal an die überwunden geglaubte »totale Institution«, als die Erving Goffmann die Heime einst charakterisierte. (6)
Auch der »Runde Tisch Pflege « kritisiert den Mangel an »verbindlichen Leitlinien zum Thema Pflegedokumentation« und schlägt daher vor, die Erstellung eines »Expertenstandards Dokumentation des Pflegeprozesses« zu prüfen.

\section{Administrative Wasserköpfe}

Überflüssige bürokratische Anforderungen werden den Heimen nicht nur von außen übergestülpt, sondern sind teilweise auch hausgemacht. Gerade große Organisationen tendieren zu bürokratischen Strukturen und damit einhergehender Schwerfälligkeit. So bestehen in manchen Einrichtungen administrative Wasserköpfe, deren Notwendigkeit für die Erbringung der Dienstleistung Pflege nicht erkenntlich ist. Sie entwickeln jedoch oft eine Eigendynamik, durch die erhebliche personelle und zeitliche Ressourcen gebunden werden. Auch entstehen dadurch meist langwierige und für die Mitarbeiter somit oft frustrierende Entscheidungsprozesse, welche die Arbeit nicht fördern, sondern behindern. Überflüssige Hierarchieebenen kosten zudem Geld, das besser in Pflege- und Betreuungskräfte investiert wäre. Träger und Einrichtungen sollten sich daher selbstkritisch fragen, ob die interne Struktur und Ablauforganisation für eine optimale Erbringung ihrer Leistungen tatsächlich dienlich ist.

Damit sich die Heime wieder auf ihre eigentliche Aufgaben konzentrieren können, muss der bestehenden Bürokratisierung und Überregulierung der Heime entgegengewirkt werden. Externe Kontrollen sind unverzichtbar, doch es bedarf dringend einer Regulierung der Aufgaben der Aufsichtsbehörden. Alle Vorschriften sollten dahingehend überprüft werden, ob sie tatsächlich zu einer Verbesserung der Lebenssituation der Heimbewohner beitragen oder ob es sich dabei um überflüssige bürokratische Anforderungen handelt. Zugleich sind aber auch alle Träger und Einrichtungen aufgefordert, ihre betriebsinterne Organisation zu überprüfen und gegebenenfalls zu verschlanken.

\section{Anmerkungen}

(1) Verband Deutscher Alten- und Behindertenhilfe e. V., Agenda »Weniger Bürokratie - mehr Pflege!«, Essen/Hannover, 2003, S. 3.

(2) Deutsches Zentrum für Altersfragen, Geschäftsstelle Runder Tisch Pflege, Arbeitsgruppe III Entbürokratisierung, 2005 (als Download im Internet http://www.dza.de erhältlich).

(3) Forschungsgesellschaft für Gerontologie e. V., Entbürokratisierungspotenziale in stationären Einrichtungen der Altenpflege, Dortmund, 2003, S. 9.

(4) Michael Graber-Dünow, Bürokratie - und kein Ende, in: Dr. med. Mabuse, Nr. 155, Mai/Juni 2005, S. 37.

(5) Michael Graber-Dünow, Bewohnerorientierung als Qualitätsmerkmal, in: Altenheim 7/2004, S. 34 ff.

(6) Erving Goffmann, Asyle, Über die soziale Situation psychiatrischer Patienten und anderer Insassen, Frankfurt am Main, 1973, S. 33 . 